\title{
POTENTIAL SPACE APPLICATIONS FOR BODY-CENTRIC WIRELESS AND E-TEXTILE ANTENNAS
}

\author{
T.F. Kennedy, P.W. Fink, A.W. Chu, G.F. Studor \\ NASA Johnson Space Center, Houston, TX, USA.
}

Keywords: e-textiles, wireless, equiangular spiral, smart antenna, hybrid coupler

\begin{abstract}
Space applications of body-centric wireless and e-textile antennas are numerous. Along with the traditional data sources, such as voice, tracking, and video, new data sources including biotelemetry, high-rate video and data, detailed tracking information and status of handheld tools, devices and on-body inventories have brought about a need for increased performance from antenna systems. E-textile antennas, which typically entail lighter weights and highly conformal properties, show great potential for the next generation of space antenna systems. Antennas made from textiles, however, must be rugged and robust for use in space environments.
\end{abstract}

\section{Introduction}

With wireless voice, video, and data sources becoming ubiquitous, the ability to integrate antennas and other radio frequency (RF) devices into body-centric systems is increasingly important. Space environment benefits of bodycentric wireless communications are numerous, particularly in the context of long duration Lunar and Martian outposts that are in planning stages at several space agencies around the world. Since crew time for such missions is a scarce commodity, seamless integration of body-centric wireless from various sources is paramount. Along with the traditional consumer applications including mobile computing and communications, body-centric space applications call for the integration of biotelemetry, detailed tracking information and status of handheld tools, devices and on-body inventories. Etextile antennas show promise in enabling such an integrated wireless platform, but a successful implementation requires overcoming some key challenges.

Operational conditions for space applications of body-centric wireless systems are often such that antennas are a limiting factor in wireless performance. Additionally, the changing antenna platform, i.e. the dynamic wearer, can detune and alter the radiation characteristics of e-textile antennas, making antenna element selection and design challenging. Antenna designs and systems that offer moderate-to-high bandwidths, perform well with flexure, and are electronically reconfigurable are ideally suited to such applications.

In creating moderate-to-high bandwidth e-textile antennas and RF devices, researchers are faced with the challenge of transferring conventional and novel designs to textiles. To create the conductive patterns of conventional antennas, wearable antennas [1-6] have been constructed using woven conductive fabrics, by bonding copper conductors directly to a fabric substrate, or with embroidery, often involving either simple conductive patterns or labor intensive processes for more complex patterns. With e-textile antennas, there is also the additional challenge of creating reliable interfaces between the textile portion of the system and the conventional hardware. Addressing these limitations is critical to the continued development and acceptance of fabric-based circuits for body-centric wireless applications.

Here we present several examples of e-textile antennas and RF devices, created using a NASA-developed process, that overcome several of these limitations. The design and performance of an equiangular spiral, microstrip antenna array, and a hybrid coupler are considered, with the e-textile devices showing comparable performance to like designs using conventional materials. Preliminary performance characterization of the antennas as they undergo flexure, as presented here and in [7-8] indicates that good antenna performance can be maintained, and that a combination of antenna design and placement are useful in creating robust designs. Additionally, through utilization of modern smart antenna techniques, even greater flexibility is permitted since antenna performance can be adjusted in real-time to compensate for the antenna's changing environment.

\section{Material Considerations}

To construct the conducting parts of an antenna using textiles, there are a variety of conducting fabric materials available. For all the e-textile devices used in this paper, the woven conductive fabric, Nora, is used. This fabric was obtained from Shieldex Trading, Inc. Nora has a nylon base and is plated with silver, copper, and nickel. Its thickness is 0.06 $\mathrm{mm}$ with a manufacturer's surface resistivity specification of $0.03 \Omega$ /square, which is excellent for creating efficient 
antennas and RF circuits at traditional wireless communication frequencies.

Several different substrate materials are used, including Nomex and a polyester cloth obtained from a retail fabric store. The polyester cloth was selected to serve as the base for the conductive pattern of the equiangular spiral. In lieu of a material pedigree and property values, measurements were made to obtain an estimate of the material properties of the cloth. Fabric measurements were conducted between $0.82-$ 4.0 GHz using a Damaskos, Inc. Model 08 Thin Sheet Dielectric Tester with Damaskos "Cavity ${ }^{\mathrm{TM}}$ " software on an Agilent E8362B network analyzer. This measurement configuration uses a rectangular cavity to measure the inplane component of $\varepsilon$ and $\tan \delta$ for thin $(<3 \mathrm{~mm})$ samples with $\varepsilon<10$. The polyester cloth was measured in the Damaskos cavity with the following results.

\begin{tabular}{|l|l|l|l|l|}
\hline material & thickness & $\varepsilon^{\prime}$ & $\varepsilon^{\prime \prime}$ & $\tan \delta$ \\
\hline polyester cloth & $0.43 \mathrm{~mm}$ & 1.99 & 0.138 & 0.070 \\
\hline
\end{tabular}

Table 1. Electrical properties of polyester cloth.

The Damaskos post-processing algorithm requires the user to input the sample material thickness. Since an accurate measurement of the material thickness was difficult, a bound on the permittivity error is obtained by entering a range of thicknesses centered at the nominal value, as shown below.

\begin{tabular}{|c|c|c|c|c|}
\hline$\Delta$ thickness & thickness & $\varepsilon^{\prime}$ & $\varepsilon^{\prime \prime}$ & $\tan \delta$ \\
\hline$-0.13 \mathrm{~mm}$ & $0.3 \mathrm{~mm}$ & 2.4 & 0.196 & 0.082 \\
\hline$+0.13 \mathrm{~mm}$ & $0.56 \mathrm{~mm}$ & 1.8 & 0.107 & 0.061 \\
\hline
\end{tabular}

Table 2. Upper and lower bounds on the electrical properties of the polyester cloth.

This fabric was found to be considerably more lossy than other fabrics tested in this study. Whereas we found this material to significantly degrade performance when used as a microstrip substrate, it does not noticeably affect the performance of the spiral antenna as will be demonstrated.

Characterization of the Nomex fabric was performed in a similar manner. Two different thickness Nomex felts were used and their properties measured using the cavity perturbation technique described previously with the results shown in Table 3 . Nomex felts have electrical properties similar to that of air, which along with their well known suitability for rugged environments, makes them ideal for use in moderate-to-high bandwidth antennas.

\begin{tabular}{|c|c|c|c|c|}
\hline material & thickness & $\varepsilon^{\prime}$ & $\varepsilon^{\prime \prime}$ & $\tan \delta$ \\
\hline Nomex & $1.57 \mathrm{~mm}$ & 1.39 & 0.013 & 0.010 \\
\hline Nomex & $6.35 \mathrm{~mm}$ & 1.18 & 0.005 & 0.004 \\
\hline
\end{tabular}

Table 3. Electrical properties for the Nomex felts as measured using the Damaskos thin sheet tester.

\section{E-Textile Designs}

To demonstrate an e-textile antenna with a complex conductive pattern and high bandwidth, a fabric equiangular spiral antenna was constructed. The two arms, made with the Nora conducting fabric, of the spiral were applied to the polyester cloth in a semi-automated art-to-part process. An RG-178 coaxial cable was used to feed the antenna with the outer conductor soldered to one spiral arm. The inner conductor of the coaxial cable was soldered to the opposing spiral arm, and a short length of dummy cable was also soldered to this opposing arm to maintain symmetry as demonstrated by Dyson [9]. For comparison, a similar antenna was constructed using etched copper spiral arms on a $1.52 \mathrm{~mm}$ thick Rogers RT/Duroid 5880 substrate. Both the conventional and e-textile equiangular spirals are shown in Figure 1.

VSWR for the antennas was measured with an HP 8510 network analyzer and simulated using EIGER with PEC elements [10-11]. Figure 2 shows a comparison of measured and simulated VSWRs with the reference plane at the antenna feed. Similar input responses are observed for both the conventional and e-textile spirals. In fact, the e-textile response is bounded by the conventional and simulated spiral VSWRs.

Figure 3 shows right-hand circularly polarized gain plots at 2 and $4 \mathrm{GHz}$, with good agreement between e-textile and copper-based antennas. Similarly a plot of the axial ratio in Figure 4 shows excellent agreement between the two antennas. Measurement and simulation reference planes were located at the antenna feed, and the impedance mismatch for the conventional copper antenna was used to offset EIGER directivity patterns. 


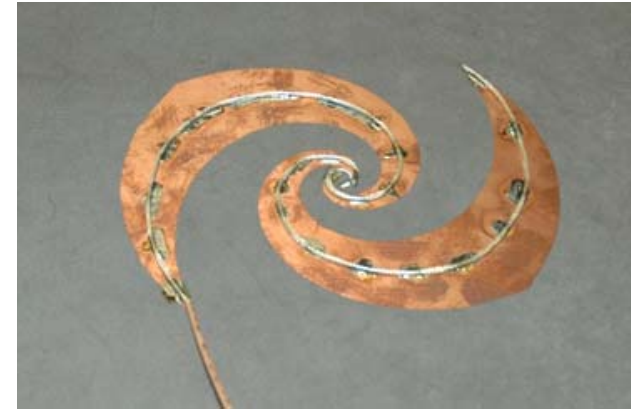

(a)

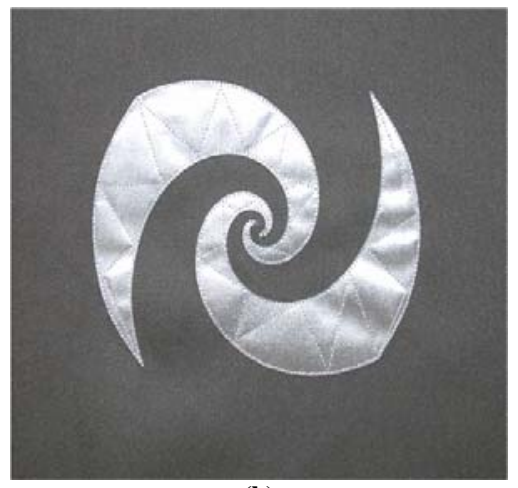

(b)

Figure 1. Conventional equiangular spiral and e-textile equiangular spiral designed for 2 - 4GHz operation.

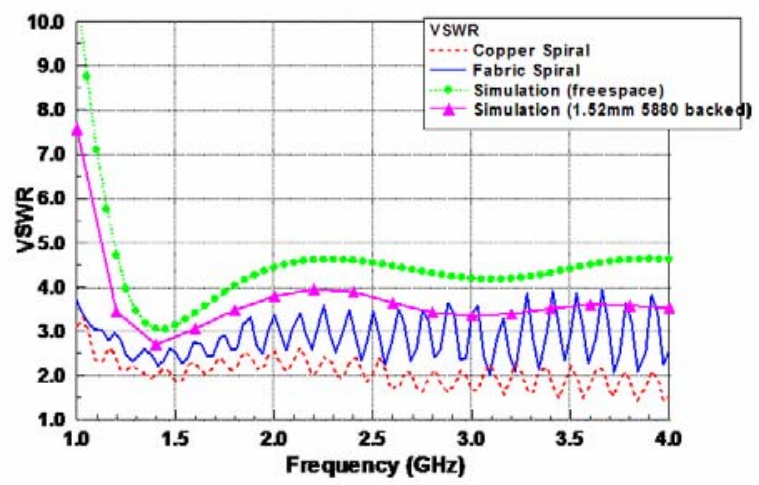

Figure 2. VSWR for conventional and e-textile based equiangular spirals.

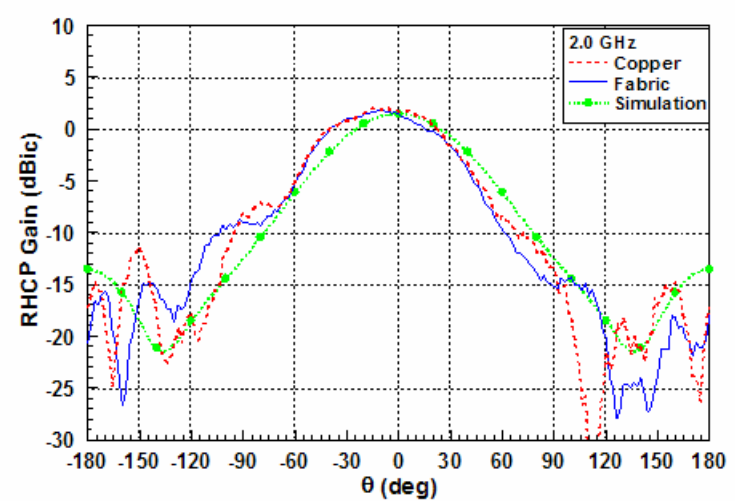

(a)

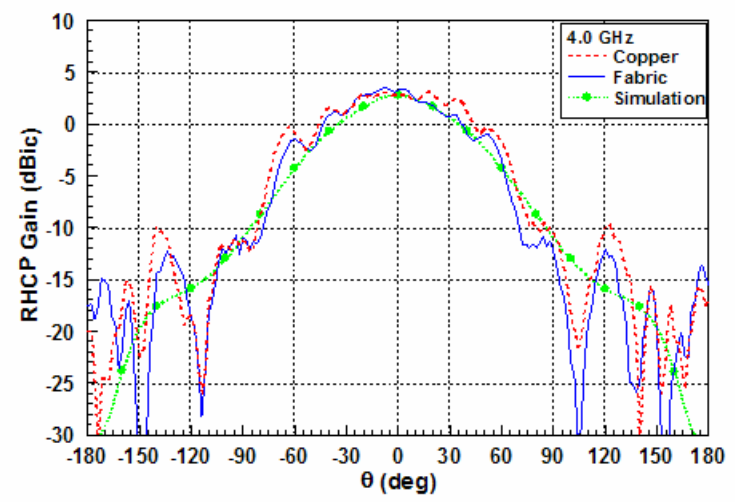

(b)

Figure 3. Gain patterns (RCP) for the conventional and e-textile based equiangular spirals at 2 and $4 \mathrm{GHz}$.

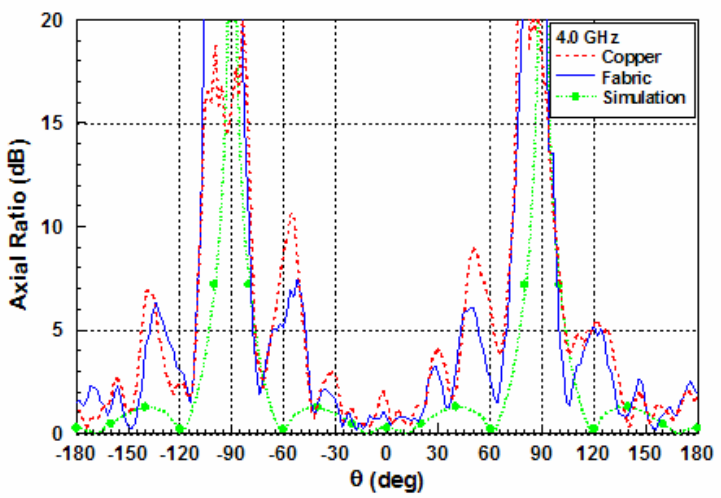

Figure 4. Axial ratio for the conventional and e-textile based equiangular spirals at $2 \mathrm{GHz}$.

In addition to single antenna elements, on-body antenna systems are likely to benefit from the addition of antenna arrays, particularly smart antenna systems since these are designed to be highly adaptable to a dynamic communications channel. To support such systems, antenna arrays must 
perform well when worn by an individual, whose movements will inevitably cause the antennas to flex.

Several authors have studied the effects of antenna flexure on the input impedance and radiation characteristics of e-textile microstrip antennas [7-8]. Results showed slight detuning of the antenna elements; however, with a sufficiently large impedance bandwidth, good antenna performance was maintained. Additionally, by bending the antennas outward; i.e. such that the linear dimensions of the patch antenna increase, an increase in beamwidth and reduction in gain was observed.

In a single antenna communication system, such changes in the pattern characteristics of an antenna with flexure and wrinkling must be accepted; however, smart antenna arrays can correct some of the deficiencies that occur under such circumstances. Antenna performance should not, however, be compromised significantly. To demonstrate e-textile antennas in an array environment, an eight-element e-textile microstrip patch array is constructed as shown in Figure 5. The array is arranged in a triangular lattice with an antenna spacing of $0.6 \lambda_{0}$. Each patch is square, is $4.85 \mathrm{~cm}$ on an edge, and is constructed using the process described previously for the e-textile equiangular spiral. Instead of the polyester substrate, a grounded (Nora) $6.35 \mathrm{~mm}$ thick Nomex $\left(\varepsilon_{\mathrm{r}}=\right.$ 1.18) substrate is used. The array is designed to cover the 2.4 GHz ISM (industrial, scientific and medical) band. Individual elements of the e-textile implementation of this array are shown in Figure 5(b) with numbers adjacent to each element for ease of discussion.

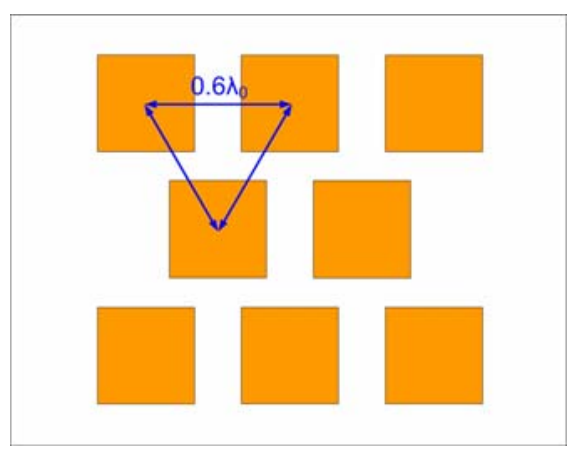

(a)

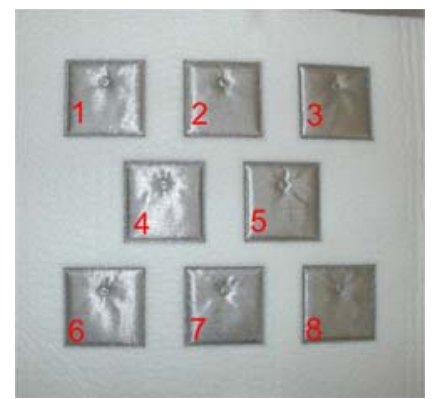

(b)

Figure 5. Eight-element patch antenna array and its implementation in fabric.

A plot of the input reflection characteristics is shown in Figure 6 for elements 1,2, and 5 of the e-textile array. Due to the symmetry of the array these three antenna elements are representative of the other antennas within the array. Note that each antenna in the array gives approximately $6.5 \%$ bandwidth which is sufficient to cover the $2.4-2.483 \mathrm{GHz}$ ISM band.

To demonstrate antenna performance in a dynamic environment, the antenna array shown in Figure 5 was bent both inward and outward on a $14.6 \mathrm{~cm}$ radius of curvature. The antenna array bent inward is shown in Figure 7. The resulting input responses for both configurations are plotted in Figure 6 with the original response of the planar array. Note a significant amount of detuning is not observed for the flexed cases relative to the individual element responses of the planar antenna array. In fact, the antennas provide the necessary bandwidth to cover the $2.4 \mathrm{GHz}$ ISM band in all configurations. Hence, under a moderate amount of static and dynamic flexure, which occurs naturally in a body-centric environment, good performance is expected. Robust performance with a moderate amount of wrinkling is also expected since in Figure 7(a) each patch conductor clearly exhibits variable amounts of wrinkling. 

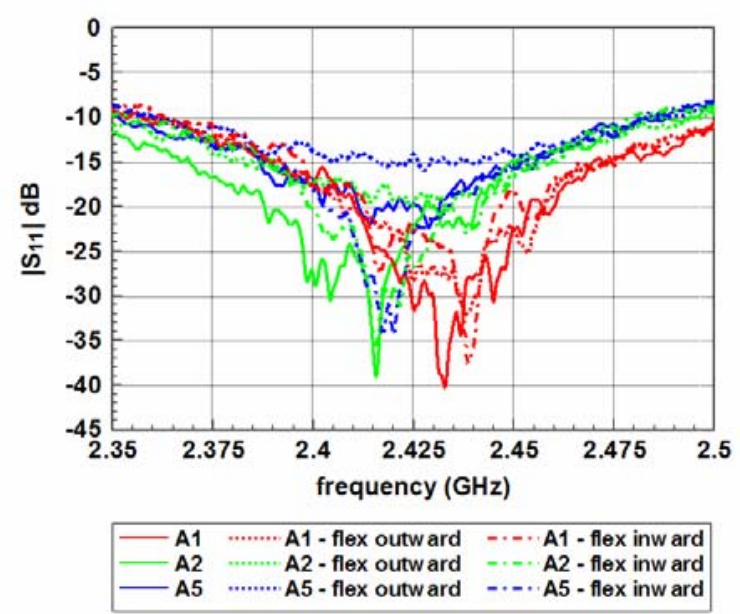

Figure 6. Reflection characteristics of the fabric antennas with and without flexure.

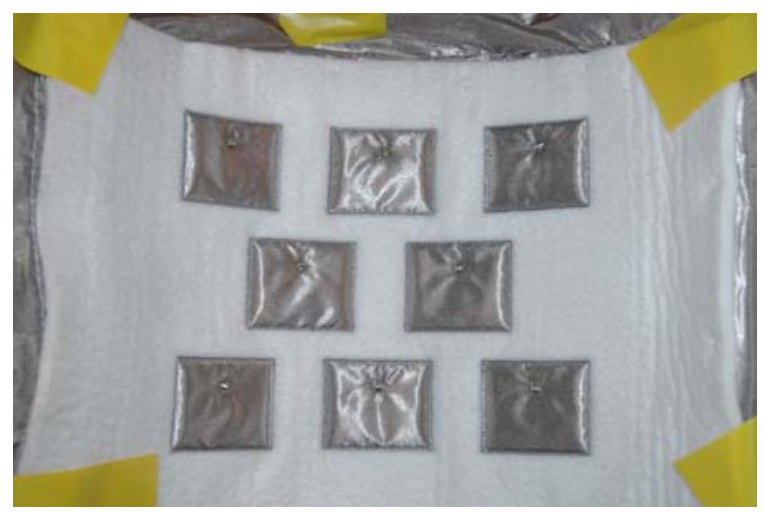

(a)

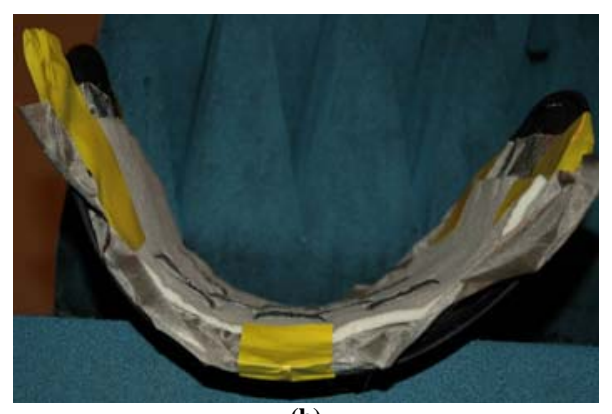

(b)

Figure 7. E-Textile array bent inward on a $14.6 \mathrm{~cm}$ radius of curvature.

An additional challenge that has been observed with e-textiles is system integration. Reliable integration of e-textile antennas with other RF components is essential to the successful implementation of an e-textile antenna system, with the ideal method being the use of e-textile RF components. A variety of standard RF components are amenable to such implementations with good performance achievable. In space applications, where circularly polarized antennas are often utilized, a common RF element to generate this polarization is a quadrature hybrid coupler. In fact, integration of such a coupler with an e-textile antenna is highly desirable for the reduction of weight in an antenna system. Good performance, however, is necessary since low circuit loss and good axial ratio performance are critical in space antenna systems.

An example coupler has been constructed with textiles, and is shown in Figure 8. All four ports are labelled in the figure and Nora is again used with a Nomex $(1.57 \mathrm{~mm})$ substrate for construction of the hybrid. Performance of this coupler is comparable to conventional ones, and the return loss at port 1 , the input port, is shown in Figure 9. From the figure, the impedance bandwidth of this coupler is $53 \%$. Coupling of port 1 to ports 2 and 4 is shown in Figure 10(a) with the phase difference shown in Figure 10(b). Note that a nearly equal power split is attained between ports 2 and 4 as well as a near $90^{\circ}\left( \pm 5^{\circ}\right)$ phase shift between these two ports over a $20 \%$ bandwidth. The insertion loss for the coupler is $0.9 \mathrm{~dB}$ at 2 $\mathrm{GHz}$, which is only slightly worse than that attained using commercially available couplers constructed from conventional materials. It is expected that an improvement in connector integration, optimized transmission line width, and improved techniques for cutting the conducting fabric, in this case Nora, should enable e-textile couplers that rival the performance of conventional couplers.

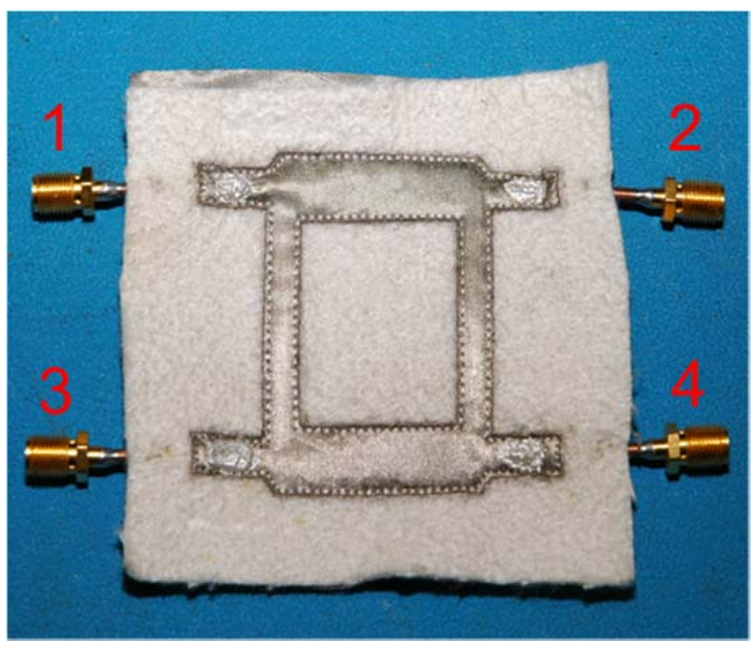

Figure 8. E-Textile quadrature hybrid coupler. 


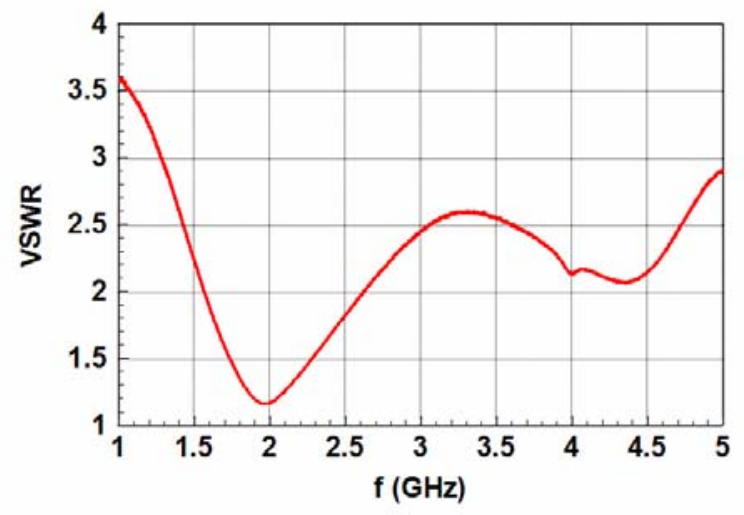

Figure 9. VSWR for Port 1 of the e-textile hybrid coupler.

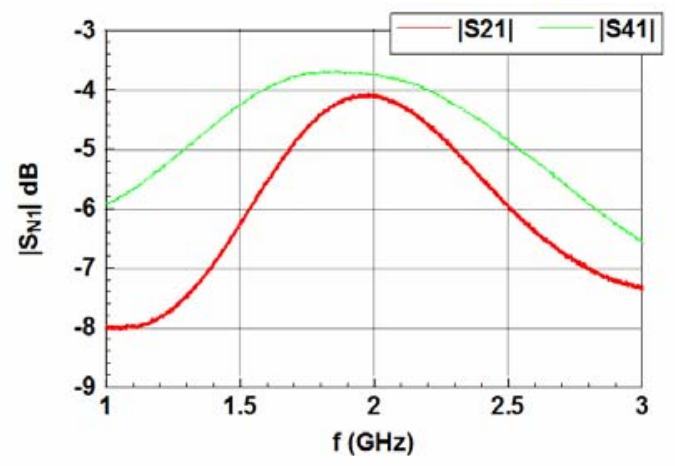

(a)

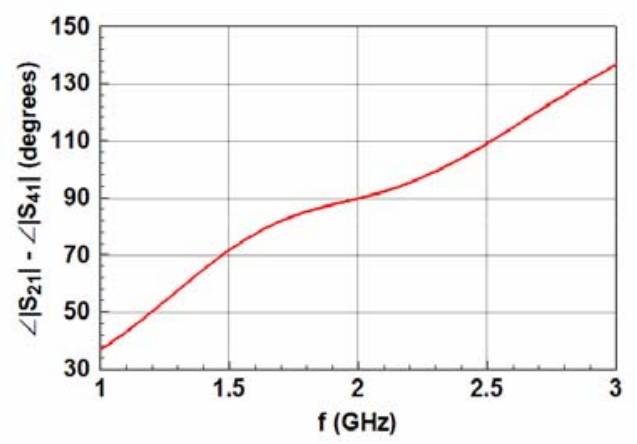

Figure 10. Magnitude of signal at the coupled ports (a) and phase difference at the coupled ports (b).

\section{Conclusions}

Several e-textile antenna and RF circuit designs were constructed and shown to have comparable performance to conventional copper-clad laminate designs. Such performance enables e-textile systems to provide all of the benefits offered by conventional antenna systems with the added benefits provided by textiles. Added flexibility of etextile designs make them ideal for space applications where light-weight, robust systems are crucial. Additionally, the added capability that can be realized with less weight enables utilization of numerous other data sources, such as high-rate video, biotelemetry, and electronic on-body inventories.

\section{Acknowledgements}

The authors would like to thank Wayne Cope, Romeo Sanchez, and Robert Trevino for their hard work and support in this effort.

\section{References}

[1] Y. Ouyang, E. Karayianni, W.J. Chappell, "Effect of fabric patterns on electrotextile patch antennas," IEEE Antennas \& Propagat. Int. Symposium, July 2005, Vol. 2B, pp. 246-249.

[2] P. Salonen, M. Keskilammi, J. Rantanen, L. Sydanheimo, "A novel Bluetooth antenna on flexible substrate for smart clothing," IEEE International Conference on Systems, Man, and Cybernetics, October 2001, Vol. 2, pp. 789-794.

[3] P. Salonen, J. Kim, Y. Rahmat-Samii, "Dual-band Eshaped patch wearable textile antenna," IEEE Antennas \& Propagat. Int. Symposium, July 2005, Vol. 1A, pp. 466-469.

[4] P.J. Massey, "GSM fabric antenna for mobile phones integrated within clothing," IEEE Antennas \& Propagat. Int. Symposium, July 2001, Vol. 3, pp. 452-455.

[5] M. Klemm, I. Locher, and G. Tröster, "A Novel Circularly Polarized Textile Antenna for Wearable Applications," Proc. of 7th European Microwave Week, October 2004, pp. 137-140.

[6] E.R. Post, M. Orth, P.R. Russo, N. Gershenfeld, "Ebroidery: Design and fabrication of textile-based computing," IBM Systems Journal, Vol. 39, Nos. 3 \& 4, 2000, pp. 840-860.

[7] I. Locher, M. Klemm, T. Kirstein, G. Troster, "Design and characterization of purely textile patch antennas," IEEE Trans. on Adv. Packaging, Vol. 29, No. 4, November 2006.

[8] A. Tronquo, H. Rogier, C. Hertleer, L. Van Langenhove, "Robust planar textile antenna for wireless body LANs operating in $2.45 \mathrm{GHz}$ ISM band," Electronics Letters, Vol. 42, No. 3, February 2006.

[9] J.D. Dyson, "The equiangular spiral antenna," IEEE Trans. Antennas and Propagation, pp. 181-187, April 1959.

[10]R.M. Sharpe, J.B. Grant, N.J. Champagne, W.A. Johnson, R.E. Jorgenson, D.R. Wilton, W.J. Brown, and J.W. Rockway, "EIGER: Electromagnetic Interactions GEneRalized," IEEE Antennas \& Propagat. Int. Symposium, 1997 Digest, vol. 4, pp. 2366-2369.

[11]W.A. Johnson, R.E. Jorgenson, L.K. Warne, J.D. Kotulski, J.B. Grant, R.M. Sharpe, N.J. Champagne, D.R. Wilton, and D.R. Jackson, "Our experiences with object oriented design, FORTRAN 90, and massively parallel 
computations," 1998 Digest USNC/URSI National Radio Science Meeting, June 21-26, 1998, Atlanta, Georgia, p.308. 


\section{Potential Space Applications for Body-Centric Wireless and E-Textile Antennas}

Timothy F. Kennedy, Patrick W. Fink, Andrew W. Chu, George F. Studor

NASA Johnson Space Center

Houston, TX 77058

Space environment benefits of body-centric wireless communications are numerous, particularly in the context of long duration Lunar and Martian outposts that are in planning stages at several space agencies around the world. Since crew time for such missions is a scarce commodity, seamless integration of body-centric wireless from various sources is paramount. Sources include traditional data, such as audio, video, tracking, and biotelemetry. Newer data sources include positioning, orientation, and status of handheld tools and devices, as well as management and status of on-body inventories. In addition to offering lighter weight and flexibility, performance benefits of e-textile antennas are anticipated due to advantageous use of the body's surface area.

In creating e-textile antennas and RF devices, researchers are faced with the challenge of transferring conventional and novel designs to textiles. Lack of impedance control, limited conductivity, and the inability to automatically create intricate designs are examples of limitations frequently attributed to e-textiles. Reliable interfaces between e-textiles and conventional hardware also represent significant challenges. Addressing these limitations is critical to the continued development and acceptance of fabric-based circuits for body-centric wireless applications. Here we present several examples of e-textile antennas and RF devices, created using a NASA-developed process, that overcome several of these limitations. The design and performance of an equiangular spiral, miniaturized spiral-loaded slot antenna, and a hybrid coupler are considered, with the e-textile devices showing comparable performance to like designs using conventional materials.

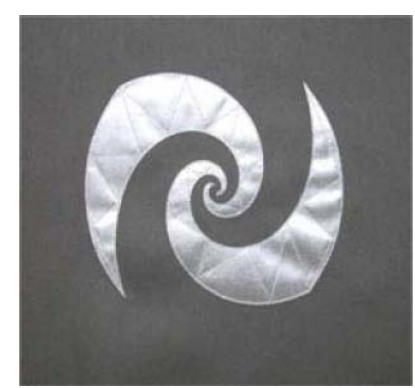

(a)

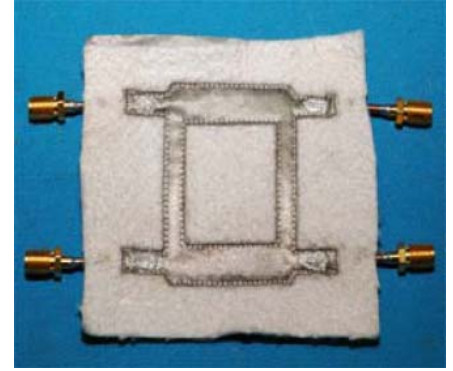

(b)

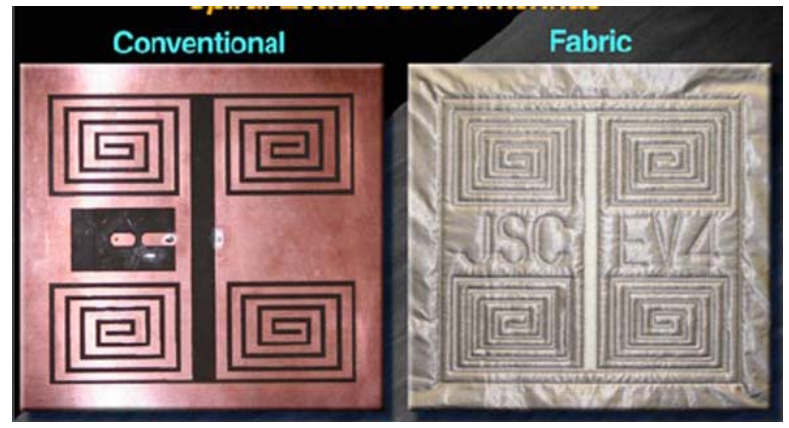

(c)

Figure 1(a) E-textile equiangular spiral. (b) Quadrature hybrid. (c) Miniaturized spiral-loaded slot antenna. 